\title{
PHOTORESIST-DERIVED POROUS CARBON FOR INTEGRATED ON-CHIP ENERGY STORAGE
}

\author{
B.I. Hsia, M. Vincent, M.S. Kim, C. Carraro, and R. Maboudian* \\ Department of Chemical and Biomolecular Engineering, University of California, Berkeley, Berkeley, CA
}

\begin{abstract}
We have developed a facile, and scalable fabrication process for high surface area porous carbon for microsupercapacitor applications. A porous carbon film is synthesized by pyrolysis of commercial SPR-220 photoresist in an $\mathrm{H}_{2} / \mathrm{Ar}$ mixture and tested for specific capacitance. A capacitance of about $2 \mathrm{mF} / \mathrm{cm}^{2}$ was achieved, matching or exceeding numerous other proposed microsupercapacitor technologies. The material was also demonstrated to be robust under cycling in aqueous electrolyte, retaining $>92 \%$ of its original capacitance after $10^{6}$ cycles.
\end{abstract}

\section{INTRODUCTION}

The increasing technological demand for autonomous sensing platforms necessitates the development of integrated energy storage for powering sensors, actuators, and communications microsystems. Supercapacitors, one potential candidate for on-chip energy storage, store charge electrostatically at the electrode/electrolyte interface, in contrast batteries, which rely on electrochemical energy storage in the bulk electrode. Because of this interfacial charge storage mechanism, supercapacitors charge and discharge much more quickly (in seconds vs. in minutes or hours for batteries) and have much longer cycle lifetime $\left(10^{6} \mathrm{vs}\right.$. $10^{3}$ cycles for batteries), but they require high surface area electrodes to achieve good energy density [1]. Because of this high surface area requirement, activated carbon is a popular material choice for supercapacitor electrodes [2]. However, for integrated, planar, on-chip supercapacitors, activated carbon presents many fabrication challenges including patterning and placement of electrodes [3].

For on-chip supercapacitor applications, an effective approach is utilization of photoresist-derived carbon since photoresist processing is well-established in microfabrication methodologies. However, prior reports on photoresist-derived carbon from AZ4300 and microstructured SU-8 resists show relatively low capacitance values unless the electrode material is chemically activated or decorated with electroactive materials [4] [5] [6] [7]. In this manuscript, we demonstrate that the pyrolysis of another readily available photoresist, SPR-220, yields a highly capacitive film without any additional treatment. The resultant capacitance from our procedure is also competitive with other proposed carbon-based microsupercapacitor electrode materials including inkjet-printed carbon [3], carbide-derived carbon [8], and carbon nanotubes [9].

\section{METHODS}

The fabrication procedure, inspired by Ref. [4], is illustrated in Fig. 1. A commercially available photoresist, SPR-220, is spin coated onto a substrate, and soft baked at $115^{\circ} \mathrm{C}$ for 3 minutes. Standard lithographic patterning can then be used to pattern the photoresist if desired. The photoresist is then annealed at $300^{\circ} \mathrm{C}$ for 30 minutes in 1 Torr of Ar to drive off residual solvent. This preanneal is followed by a $900^{\circ} \mathrm{C}$ pyrolysis in 1 Torr of a $10 \% \mathrm{H}_{2} / 90 \%$ Ar mixture. After 1 hour, the sample is allowed to cool to room temperature in the mixed gas environment.

\section{RESULTS \& DISCUSSION}

Figure 2 shows an optical image of a film patterned into an interdigitated comb electrode configuration on a $\mathrm{Si}$ substrate, before and after pyrolysis. While the lateral dimensions are approximately maintained after annealing, the film thickness is reduced by about a factor of 5 after annealing, from $10 \mu \mathrm{m}$ to 2 $\mu \mathrm{m}$, as determined by profilometry.

Figure 3 shows the Raman spectra of unannealed and annealed photoresist in comparison to commercial activated carbon. Both the activated carbon and annealed photoresist show the characteristic peaks of graphitic carbon, the D- and G- peaks, at about $1350 \mathrm{~cm}^{-1}$ and $1600 \mathrm{~cm}^{-1}$ respectively, confirming the carbonaceous nature of the fabricated carbon film.

The capacitance of a $2 \mu \mathrm{m}$ thick porous carbon film was measured via cyclic voltammetry $(\mathrm{CV})$ in a $3.5 \mathrm{M} \mathrm{KCl}$ solution in a three-probe configuration with a $\mathrm{Pt}$ counter electrode and an $\mathrm{Ag} / \mathrm{AgCl}$ reference electrode, and the result is presented in Fig. 4. The CV curve shape is semi-rectangular for biases between 0 and $+0.7 \mathrm{~V}$ indicating ideal capacitive behavior. The sharp current increase for bias $<+0.8 \mathrm{~V}$ and $<-0.2 \mathrm{~V}$ likely stem from irreversible processes at the electrode surface. The other small irregularities likely stem from pseudocapacitive reactions at interfacial functional groups [10]. Specific capacitance is calculated via Eq. 1, where $I$ is the current taken at a midpoint voltage, $+0.25 \mathrm{~V}, d V / d t$ is the voltage sweep rate, and $A$ is the projected area of the film.

$$
C=\frac{I}{d V / d t} \frac{1}{A}
$$

The measured capacitance is measured to be about $2 \mathrm{mF} / \mathrm{cm}^{2}$ for all scan rates, matching or exceeding the capacitance for inkjetprinted activated carbon [3] and carbon nanotubes [9]. Carbidederived carbon, with an areal capacitance of $30 \mathrm{mF} / \mathrm{cm}^{2}$ for $2 \mu \mathrm{m}$ films, has demonstrated greater capacitance values, but requires a lengthier fabrication including high temperature annealing in a chlorine environment and carbon etching [8]. Additionally, we predict that thicker photoresist films may give increased areal capacitance, and that chemical treatments may improve porosity and pseudocapacitance [6] [10]. Previous tests on annealed AZ4300 and microstructured SU-8 photoresists gave capacitances of $8 \mu \mathrm{F} / \mathrm{cm}^{2}$ [4] and $<60 \mu \mathrm{F} / \mathrm{cm}^{2}$ [6] respectively, indicating that our process likely yields a much more porous, high surface area film, resulting in a capacitance improvement of two orders of magnitude. To demonstrate the robustness of the film, a cycle lifetime test was also performed that showed $<8 \%$ drop in capacitance over one million charge/discharge cycles, as shown in Fig. 5. This compares favorably with Ref. [6], where a chemically activated pyrolyzed carbon electrode showed a $>12 \%$ drop in capacitance over one thousand cycles.

In summary, a simple, easily integratable process has been developed for fabrication of porous carbon electrodes via a photoresist annealing process. These electrodes exhibit a high specific capacitance and a long cycle lifetime in aqueous electrolyte. A planar, integrated, two-electrode device is easily fabricated via standard photolithographic techniques. Future work will focus on chemical pretreatments to increase specific surface area and pseudocapacitance, and device fabrication and characterization in a variety of electrolytes. 


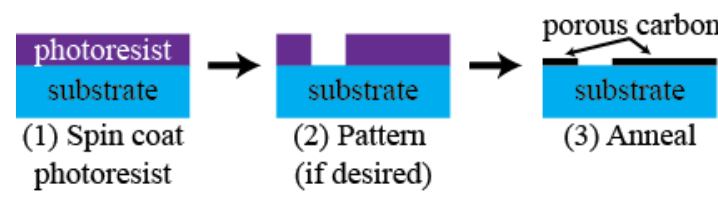

Figure 1: Fabrication schematic

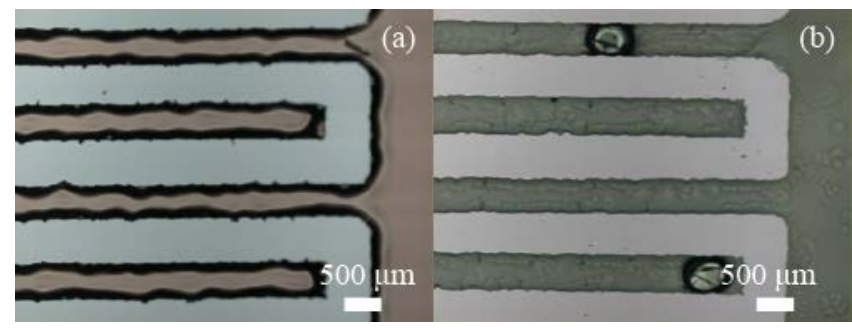

Figure 2: Optical image of comb electrode pattern (a) before (photoresist) and (b) after annealing (porous carbon). Lateral size of patterned structures is not significantly altered by annealing.

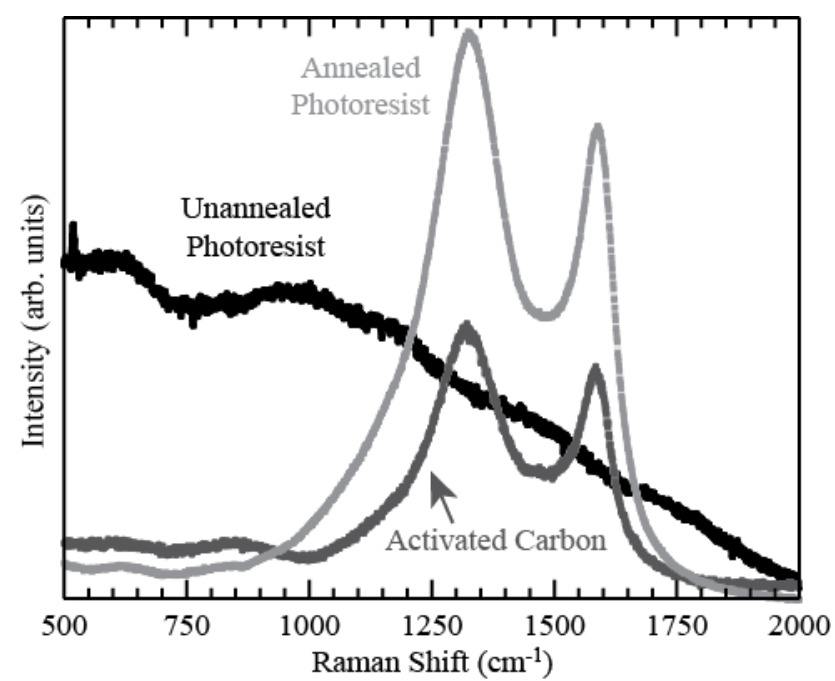

Figure 3: Raman spectra of unannealed photoresist, annealed photoresist, and activated carbon.

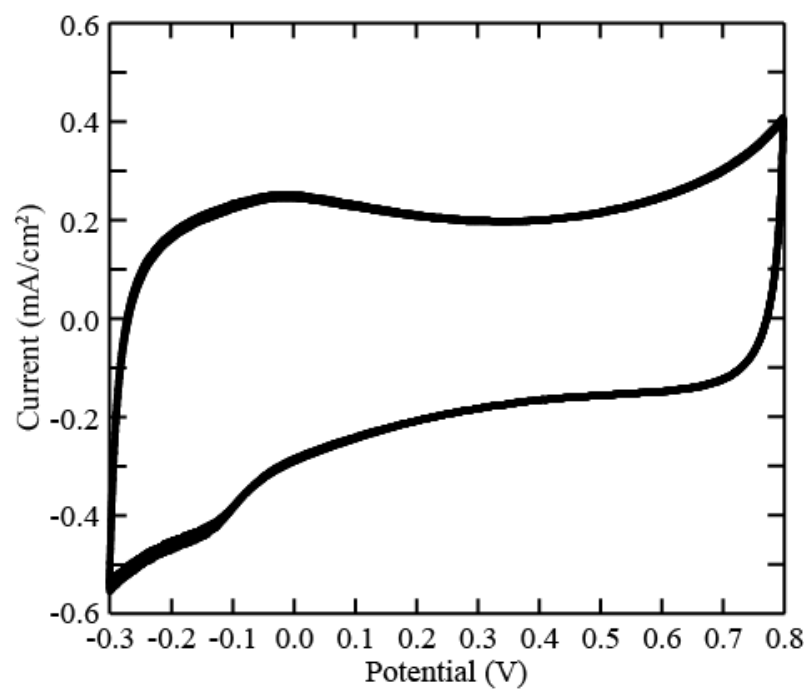

Figure 4: Cyclic voltammogram of $2 \mu \mathrm{m}$ thick annealed photoresist film in $3.5 \mathrm{M} \mathrm{KCl}$. Scan rate is $100 \mathrm{mV} / \mathrm{s}$.

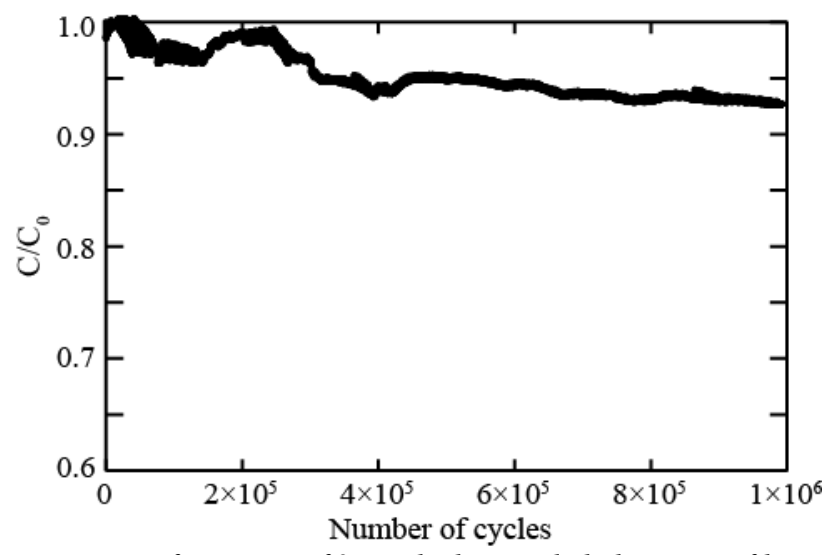

Figure 5: Lifetime test of $2 \mu \mathrm{m}$ thick annealed photoresist film in $3.5 \mathrm{M} \mathrm{KCl}$. Scan rate is $5 \mathrm{~V} / \mathrm{s}$ and potential window is $-150 \mathrm{mV}$ to $150 \mathrm{mV}$.

\section{REFERENCES}

[1] P. Simon and Y. Gogotsi, "Materials for Electrochemical Capacitors," Nature Mat., vol. 7, pp. 845-854, 2008.

[2] E. Frackowiak, "Carbon materials for supercapacitor application," Physical Chemistry Chemical Physics, vol. 9, pp. 1774-1785, 2007.

[3] D. Pech, M. Brunet, P.-L. Taberna, P. Simon, N. Fabre, F. Mesnilgrente, V. Conedera and H. Durou, "Elaboration of a microstructured inkjet-printed carbon electrochemical capacitor," J. Power Sources, vol. 195, no. 4, p. 1266-1269, 2010.

[4] S. Ranganathan, R. McCreery, S. M. Majji and M. Madou, "Photoresist-Derived Carbon for Microelectromechanical Systems and Electrochemical Applications," J. Electrochem. Soc., vol. 147, no. 1, pp. 277-282, 2000.

[5] M. Beidaghi and C. Wang, "Micro-supercapacitors bsaed on three dimensional interdigital polypyrrole/C-MEMS electrodes," Electrochimica Acta, vol. 56, pp. 9508-9514, 2011.

[6] M. Beidaghi, W. Chen and C. Wang, "Electrochemically activated carbon micro-electrode arrays for electrochemical micro-supercapacitors," Journal of Power Sources, vol. 196, pp. 2403-2409, 2011.

[7] W. Chen, M. Beidaghi, V. Penmatsa, K. Bechtold, L. Kumari, W. Z. Li and C. Wang, "Integration of Carbon Nanotubes to C-MEMS for On-chip Supercapacitors," IEEE Transactions on Nanotechnology, vol. 9, pp. 734-740, 2010.

[8] J. Chmiola, C. Largeot, P.-L. Taberna, P. Simon and Y. Gogotsi, "Monolithic Carbide-Derived Carbon Films for Micro-Supercapacitors," Science, vol. 328, no. 5977, pp. 480483, 2010.

[9] Y. Q. Jiang, Q. Zhou and L. Lin, "Planar MEMS Supercapacitor using Carbon Nanotube Forests," Proc. IEEE Micr. Elect., pp. 587-590, 2009.

[10] E. Frackowiak and F. Beguin, "Carbon materials for the electrochemical storage of energy in capacitors," Carbon, vol. 39, pp. 937-950, 2001

\section{CONTACT}

*R. Maboudian, tel: +1-510-643-7957; maboudia@berkeley.edu 